\title{
The Diversity of Broad Emission-Line Profiles
}

\author{
Giovanna M. Stirpe \\ Osservatorio Astronomico di Bologna, Via Zamboni 33, 40126 Bologna, \\ Italy
}

Andrew Robinson

Division of Physical Sciences, University of Hertfordshire, College Lane, Hatfield, Herts $A L 109 A B, U K$

David J. Axon

Space Telescope Science Institute, 3700 San Martin Drive, Baltimore, $M D$ 21218, USA

\begin{abstract}
We present preliminary results from a study of broad-line profiles in active galaxies. A simple model in which the emissivity is a broken power-law function of radius, and the BLR clouds emit anisotropically, yields very good fits to almost all the $\mathrm{H} \alpha$ profiles in our data base.
\end{abstract}

\section{Introduction}

The profiles of broad-emission lines in active galaxies display a wide diversity in width and shape, which cannot be explained only by short-time scale variability or projection effects: the different profiles are, instead, the result of a wide range of intrinsic characteristics of the broad-line region (BLR) throughout the activegalaxy population. Understanding and classifying these characteristics should therefore provide insight on the nature and evolution of the BLR.

We have therefore started a study of $\mathrm{H} \alpha$ and $\mathrm{H} \beta$ profiles of about 100 , mainly radio-quiet, Seyfert 1 galaxies and low-redshift QSOs, in order to classify them on the basis of a simple model of the BLR. The distributions of the parameters can be used to search for inter-relations, and for relations with other physical properties of the objects (e.g., luminosity or variability behavior). We present here some preliminary results on $\mathrm{H} \alpha$.

\section{The Model}

The BLR is modeled as an isotropic sphere with a power-law velocity vs. radius distribution. The volume emissivity is also a power-law function of radius, but changes its index at some radius $r_{1}$ within the BLR. The slopes of the velocity and emissivity distributions combine to determine the curvatures of the line profiles. The break in the emissivity power-law determines the change in curvature which is normally observed between line wings and core. In order to reproduce 

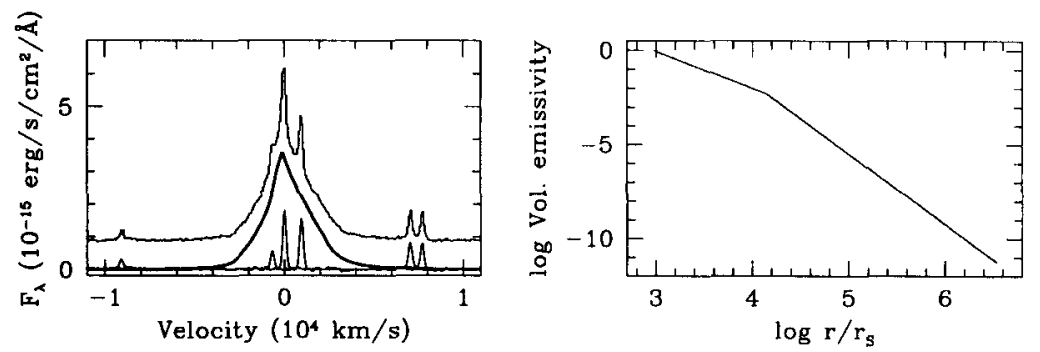

Figure 1. Left: $\mathrm{H} \alpha$ spectrum of Mrk 40, with the fitted broad and narrow components, and the residuals of the total fit. Right: The corresponding emissivity distribution (see text). The power-law indices are -1.9 and -3.8 before and after the break point, respectively.

the asymmetries of the lines, the emissivity functions have been multiplied by a factor $(1-A \cos \theta)$, where $\theta$ is the angle between the cloud velocity vector and the line of sight, and $A$ an anisotropy parameter between 1 and -1 . The anisotropy changes the curvature of the line wings and core, with different effects at positive and negative projected velocities.

The resulting profile is an analytical function of the model's 7 parameters. This function was fitted to our spectra, with additional parameters modeling the underlying continuum, the narrow lines, and the intensity and reference wavelength of the broad line. Most $\mathrm{H} \alpha$ profiles were fitted extremely well by the model, the exceptions being the few double-peaked broad lines which are usually modeled with accretion disks. An example is shown in Fig. 1.

\section{Main Results}

Assuming that the velocity field is gravitational $\left(v \propto r^{-1 / 2}\right)$, we used the fitted curvatures to derive the emissivity indices for each object. These are all negative, indicating that the emissivity is always a decreasing function of radius. The emissivity law is always flatter within and steeper beyond the break radius, and the wings are almost never logarithmic. The break point tends to occur at greater radial depth in narrow-line Seyfert 1 galaxies: the line emissivity of these objects therefore is less centrally concentrated.

There is a strong positive correlation between the anisotropy indices of the inner and outer BLR, implying that the emission in these two regions is generally beamed in the same direction. Also, the anisotropy indices are equally divided between positive and negative, reflecting the approximately equal numbers of red- and blue-asymmetric profiles. The cause of this is unknown.

The results of the fits can also be used to obtain the expected transfer functions, and thus predict how the lines would react to continuum variability.

Some open problems remain, such as explaining asymmetries which depend on projected velocity if, as variability studies imply, the velocity field is not predominantly radial. We believe, however, that steps are being made towards achieving a useful parametric description of the BLR. 